picking up another at the next station, each carriage thus slowly shifting round the line.

But any such plan would entail a fresh build of carriages; and for discontinuous carriages a plan nearly as good would be to run a railway omnibus on the rails, with a small 6 or $8 \mathrm{~h}$. p. engine all in one. This would be stopped anywhere between stations, at crossings, farmhouses, and hamlets along the line, and would serve the peasantry for going shopping, beside taking up baskets of garden produce. Passengers going a long journey would change at a main station and join the ordinary train, which would only stop about every hour at the ends of forty or fifty miles' stages. Country lines only running a train every two hours or so would be easily worked thus, the 'bus being shunted by telegraph if necessary, and the line signalled clear as usual. With double lines the 'bus would run on the goods line.

Bromley, Kent

W. M. F. Petrie

\section{A NEW KIND OF ELECTRIC REPULSION}

D. GOLDSTEIN has devoted a good part of the last ten years to an investigation of the discharge of electricity through gases, and amongst the many phenomena which he has brought to light, the one described in a memoir published in a separate form is not the least interesting and important. The facts may be stated in a few words : A negative electrode exerts a strong repulsion on the rays of the glow proceeding from itself or from another negative electrode. Before describing the experiments proving this statement, and the laws by which this phenomenon is regulated, we shall follow Dr. Goldstein in reminding the reader of a few facts connected with a discharge of electricity through gases which he will have to bear in mind.

It is well known that the negative electrode in a gas, for which Faraday's name of cathode may be conveniently used, is surrounded with a glow which expands as the pressure of the gas is reduced. We are able to distinguish four layers in this gas, though three of them only are easily recognised. As a first approximation we may assume the outline of these layers to be parallel to the outline of the electrode, though, as we shall have to mention, Dr. Goldstein has shown that this is not strictly correct.

The layer of the negative glow adjacent to the cathode is luminous, and shines in air with a yellowish-red tint. This first layer is surrounded by a second layer, which is very little luminous. This is the dark space mentioned by Mr. Crookes ; but, as Dr. Goldstein shows, it is not entirely dark, but has in air a bluish tint. We next come to the third and fourth layers, which may very well be taken as one, and which are more generally designated by means of the term, negative glow. They form the outer boundary of the luminosity surrounding the cathode. If the pressure of the gas is sufficiently reduced to enable the glow to touch the glass, it becomes phosphorescent, and only the layer of the gas immediately touching the glass causes the phosphorescence. The phosphorescence gets stronger as exhaustion proceeds; at the same time the luminosity of the glow gets weaker. The appearance and extension of the glow does not depend on the position of the anode, while the luminous positive discharge varies very much with the relative position of the electrodes, and can be made to disappear altogether by bringing the electrodes sufficiently near.

Already Plücker, and especially Hittorf, have come to the conclusion that the negative glow is propagated in rectilinear rays from the cathode, and it can further be shown that the direction of propagation is generally in a direction nearly normal to the surface of the cathode. Dr. Goldstein draws a distinction between such elements of the cathode which lie near the edge, if the surface of the cathode has edges, and elements which are removed from the edge. While those elements not near an edge only send out rays within a cone of narrow aperture in a " "A New Kind of Electric Repulsion," by Dr. E. Goldstein. (Berlin : Julius Springer, 1880.) normal direction, the edges send out rays in all directions. This difference in the behaviour of different elements of the same surface is, it appears to us, well explained by Dr. Goldstein's discovery of a repulsion between the electrode and a ray proceeding from the cathode. A little consideration will show that this repulsion will, whenever cylindrical or plane electrodes are used, be in a nearly normal direction for any part of the surface which is sufficiently removed from the edge, while near the edge the resultant repulsion will be away from the surface and from the greater angle with the normal the nearer the ray is to the edge. This would prove of course that the repulsion is not an electrostatic one, for in that case it would always be at right angles to the surface. If the exhaustion is such that the glass becomes phosphorescent, the phosphorescence, being produced by the rays proceeding from the cathode, it is clear, will form a luminous ribbon surrounding the electrode, which is a little larger than the electrode.

If now a solid body is introduced between the cathode and the glass inclosure, a shadow of this body will appear in the phosphorescent light on the glass; the formation of the shadow is a direct consequence of the rectilinear propagation of the rays.

We now proceed to describe Dr. Goldstein's experiment in its simplest form.

In a cylindrical vessel two parallel electrodes of equal length are introduced at one end, while the other end contains a third electrode which shall always form the anode. Let the pressure be such that phosphorescence appears, and let only one of the two parallel electrodes be connected with the negative pole of the coil, while the other is insulated. A shadow of this insulated wire is seen in the phosphorescent light on the glass. Now let the insulated wire be brought into metallic contact with the other electrode, and the whole appearance will change. In the phosphorescent light of the glass we shall see two dark surfaces of equal size and shape, and with distinctly marked edges. The two dark surfaces are situated in such a way that a plane which passes through the electrodes cuts them into two equal halves. They are partly bounded by straight lines, partly by two semicircular arcs.

The parts formed by straight lines are parallel to the electrodes, and of equal length; these straight lines are joined at the lower end, that is, at the free end of the electrodes, by means of a half circle, which is partially repeated at the upper end; but where the electrodes are sealed into the glass the curve is interrupted. The dark surfaces are bordered by a bright line of light. It will facilitate the understanding of the position and shape of these dark surfaces if we mention already here that they are such as would be produced if the rays emanating from each electrode, and propagated in a normal direction from it, suffer a repulsion and consequent deflection in the neighbourhood of the other electrode, so that the dark space is formed by the absence of the phosphorescent light which would be produced by the rays coming from the farther cathode.

We cannot here give the further description of shape and the measurement of the size of these dark surfaces, but at once describe their properties. In the first place the size and shape are altogether independent of the position, form, and size of the positive electrode. The relative position of the two cathodes, on the other hand, materially affects their behaviour; and Dr. Goldstein gives their shape, for instance, if, instead of being parallel, they are at right angles to each other, either in the same plane or one in front of the other. We have already stated that in the case of parallel electrodes the parts of the outline forming straight lines are of equal length with the electrodes, and hence the length of these dark surfaces increases with the length of the electrodes, but the breadth and half-circle joining the straight lines 
does not vary with the length of the clectrodes. The further removed the glass walls are from the two electrodes the greater is the width of the surfaces. All these and other properties follow at once if we consider that the luminosity is produced by the intersection of the rays proceeding from the cathodes with the glass walls. If we increase the thickness of one of the electrodes, the size of the dark surface nearest to it is increased. We now turn to the experiments which have been made in order to clear up the cause of the phenomena. By means of very ingenious experiments Dr. Goldstein proves that it is only light produced by the nearer electrode which is seen within the boundary of the dark surface, for although we have called them dark, they are only so by contrast, and they show a faint phosphorescence. Dr. Goldstein had, in a former paper, shown that when the cathode is perfectly smooth, the phosphorescent light produced by the glow shows inequalities. By twisting the aluminium electrodes he could obtain a series of spiral curves in the phosphorescent light more luminots than the rest. If one of the two cathodes is twisted in such a way and connected with the other, the spiral curves are interrupted in the dark space which is removed from the twisted electrode, but they are visible in the dark surface nearest to it.

The dark surface cannot be considered as enlarged shadows only of the electrodes, for their shape is different, but they might as regards shape be considered as shadows of the second and non-luminous layer of the negative glow. This remark we believe to be of importance, but Mr. Goldstein shows that they cannot really be such shadows, for they appear even when by an approach of the two electrodes the two non-luminous layers fuse into one and so lose their individuality.

The following experiment proves the repulsion. A metallic diaphragm is introduced between the two cathodes. A small hole is made in the diaphragm with its centre in a line joining the electrodes. Only some of the rays proceeding from each cathode can now reach the next, and consequently we observe only a small phosphorescent speck at the opposite side of the glass wall if one of the electrodes is insulated, but the dark shadow of the nearest electrode is visible in this phosphorescent speck. If now the two cathodes are joined the phosphorescent speck is seen to divide into two which separate and clearly show that the rays producing the phosphorescent light must have suffered a deflection as soon as the two cathodes were joined. Further experiments show that the deflection takes place at right angles to the surface of the electrode, and that it takes place at sensible distances from the repelling cathode, although it rapidly decreases in strength. Near the edge of a repelling cathode the repulsion does not take place in a normal direction, and Dr. Goldstein draws again a distinction between elements of a surface according as they are removed or near an edge. We believe this distinction to be unnecessary, and that all phenomena are explained by the fact that all parts of the electrode are repelling, and not only the elements nearest to the deflected ray.

Some remarkable secondary phenomena take place in a deflected system of rays. If, for instance, a system of rays forming a cone of narrow aperture passes near a second cathode, it is not only deflected but the aperture of the cone is increased. The phenomena are such as would be produced if a cathode not only repels the rays but also induces a state in the particles forming the ray such that they now repel each other. Also parts of the same cathode repel rays proceeding from other parts, and the repulsion increases the thicker the electrode. All these facts are illustrated and proved by a series of wellarranged experiments. Dr. Goldstein next examines the influence of an anode, but we shall not follow him, as it is found that the effect of an anode is exceedingly small, and most likely always produced by secondary causes.
The deflection is the same in all gases: air, hydrogen, carbonic oxide, and magnesium vapour having been tried.

The deflection is independent of the metal of which the cathode is formed; it is independent of the pressure. It is also independent of the intensity of discharge when the two electrodes are in metallic contact, so that the current is equally divided between the two cathodes. But remarkable changes take place if the current is not so equally divided. This can be done by joining the electrodes not metallically, but with a bad conductor, as for instance a moist thread. It is then found that the dark surface nearest the cathode through which the smaller discharge passes is much reduced in size, while the other dark surface is increased. It follows from experiments such as this that the repulsion does depend on the intensity of discharge, but that while a cathode through which more electricity passes more strongly repels, a ray which proceeds from such a cathode is less strongly deflected. If therefore we have seen that the dark surfaces do not vary in size, whatever the intensity of discharge, if the two poles are connected with a piece of metal; this is due to the fact that each cathode repels more strongly, but that the rays of the other electrode (owing perhaps to the greater velocity of the molecules procecding from it) are less easily deflected, and that the two effects counterbalance each other. Dr. Goldstein considers, rightly no doubt, that the shadows seen when one apparently insulated metallic body is introduced between the cathode and the glass are due to a similar repulsion, because we may consider that a small part of the discharge always passes through such a body, the glass into which the body is necessarily sealed not being an absolute non-conductor. The shape of the shadow confirms this supposition.

Dr. Goldstein has also obtained the repulsion from electrodes consisting of glass and mica, so that the metallic or non-metallic nature of the electrode does not influence the phenomena. He has also proved that the source of electricity is immaterial, as might have been expected.

Dr. Goldstein has also endeavoured to prove that the deflecting power of a cathode does not act through a solid screen, but he has chosen metallic screens for his experiment.

If the repulsion is of the nature of electric repulsion a metallic body might act as a screen, while a nonmetallic body would allow two bodies on opposite sides of it to repel each other. As it is impossible to form any idea on the cause of these phenomena unless we know whether the deflecting power is cut off by any solid body, it is much to be wished that Dr. Goldstein will repeat his experiments with non-metallic screens.

In the last part of his book Dr. Goldstein discusses various theories which might be proposed and have been proposed for the explanation of the phenomena taking place in the neighbourhood of the negative electrode. The result is that none of them are satisfactory. While this no doubt is true, Dr. Goldstein is too severe, we believe, in his criticisms of some of the suggestions which have been made, and which may, in our opinion, after all contain the germ of the true explanation, though in their present shape they may not be quite satisfactory. Some of the facts which to Dr. Goldstein are sufficient to reject a theory may, we believe, be explained without putting too great a strain on our present ideas, and sometimes we believe Dr. Goldstein to be in error, as when, for instance, he says that a body must necessarily move in a line of force. It would at least be a sad look-out for our earth if this was true, and Dr. Goldstein would in that case have occasion to study before long the electric phenomena on the surface of the sun. We will hope, for the sake or science, that both Dr. Goldstein and his molecules do not always move in lines of force, and that he will often favour us with such interesting and valuable contributions as the one before us.
ARTHUR SCHUSTER 